\title{
Erratum to: Contrasting microbial community composition and function perspective in sections of a full-scale coking wastewater treatment system
}

\author{
Shuang $\mathrm{Zhu}^{1}$ - Haizhen $\mathrm{Wu}^{2}$ - Chaohai Wei ${ }^{3} \cdot$ Lin $\mathrm{Zhou}^{1}$. \\ Junting Xie ${ }^{1}$
}

Published online: 21 January 2016

(C) Springer-Verlag Berlin Heidelberg 2016

Erratum to:Appl Microbiol Biotechnol (2016) 100:949-960

DOI 10.1007/s00253-015-7009-z

The original version of this article inadvertently contained mistake in the author's affiliations.

Original Publication:

Shuang Zhu ${ }^{1,2,3}$ \& Haizhen $\mathrm{Wu}^{1,2,3}$ Chaohai Wei ${ }^{1,2,3}$ Lin Zhou $^{1,2,3}$ Junting Xie ${ }^{1,2,3}$

Corrected Presentation:

Shuang Zhu ${ }^{1}$ Haizhen $\mathrm{Wu}^{2}$ Chaohai $\mathrm{Wei}^{3}$ Lin $Z \mathrm{Zhou}^{1}$ Junting $\mathrm{Xie}^{1}$

The online version of the original article can be found at http://dx.doi.org/ $10.1007 / \mathrm{s} 00253-015-7009-\mathrm{z}$.

\section{Chaohai Wei}

cechwei@scut.edu.cn

1 Guangdong Province Key Laboratory for Biotechnology Drug Candidates, School of Biosciences and Biopharmaceutics, Guangdong Pharmaceutical University, Guangzhou 510006, People's Republic of China

2 College of Biological Science and Engineering, South China University of Technology, Guangzhou 510006, People's Republic of China

3 School of Environment and Energy, South China University of Technology, Guangzhou 510006, People's Republic of China 